\title{
Identification and Characterization of a Novel Single Domain Antibody Against Ebola Virus
}

\author{
Rui Wang ${ }^{1,2} \cdot$ Haiwei Zhang ${ }^{1} \cdot$ Cheng Peng ${ }^{1} \cdot$ Jian Shi $^{1} \cdot$ Huajun Zhang ${ }^{1}$ (i) $\cdot$ Rui Gong $^{1,2}$ (I)
}

Received: 5 June 2021 / Accepted: 9 August 2021 / Published online: 11 October 2021

(c) Wuhan Institute of Virology, CAS 2021

\begin{abstract}
Ebola virus (EBOV) belongs to the Filoviridae family and causes severe illnesses such as hemorrhagic fever with a high mortality rate up to $90 \%$. Now two antibody drugs termed Inmazeb and Ebanga have been approved for treating EBOV infection. However, clinical studies have demonstrated that the mortality rate of the patients who received these two antibody drugs remains above $30 \%$. Therefore, novel therapeutics with better efficacy is still desired. The isolated human IgG1 constant domain 2 ( $\mathrm{CH} 2$ domain) has been proposed as a scaffold for the development of C-based single domain antibodies (C-sdAbs) as therapeutic candidates against viral infections and other diseases. Here, we screened and identified a novel C-sdAb termed M24 that targets EBOV glycoprotein (GP) from a C-sdAb phage display library. M24 neutralizes the pseudotype EBOV with $\mathrm{IC}_{50}$ of $0.8 \mathrm{nmol} / \mathrm{L}(12 \mathrm{ng} / \mathrm{mL})$ and has modest neutralizing activity against authentic EBOV. Epitope determination, including molecular docking and site mutation analysis, discloses that M24 binds to the internal fusion loop (IFL) within GP2, a transmembrane subunit of GP. Interestingly, we found that the binding of M24 to GP at pH 5.5 has dramatically decreased compared to the binding at $\mathrm{pH} 7.5$, which may lead to weak efficacy in the neutralization of authentic EBOV. Since no sdAb against EBOV infection has been reported to date, our results not only give a proof of concept that sdAbs could be utilized for the development of potential therapeutic candidates against EBOV infection, but also provide useful information for the discovery and improvement of anti-EBOV agents.
\end{abstract}

Keywords Ebola virus (EBOV) · Constant domain $2(\mathrm{CH} 2$ domain) · C-based single domain antibody (C-sdAb) · Neutralize · Fusion loop

\begin{tabular}{|c|c|c|c|}
\hline \multicolumn{3}{|c|}{ Abbreviations } & RESTV \\
\hline \multirow{2}{*}{\multicolumn{2}{|c|}{$\begin{array}{l}\text { EBOV } \\
\mathrm{CH} 2 \text { domain }\end{array}$}} & Zaire ebolavirus & RBD \\
\hline & & Constant domain 2 & MLD \\
\hline \multicolumn{2}{|c|}{ C-sdAb } & C-based single domain antibody & $\mathrm{GP}_{\mathrm{CL}}$ \\
\hline \multicolumn{2}{|c|}{ GP } & Glycoprotein & NPC1 \\
\hline \multicolumn{2}{|c|}{ IFL } & Internal fusion loop & $\mathrm{ADCC}$ \\
\hline \multicolumn{2}{|c|}{ SUDV } & Sudan ebolavirus & \\
\hline \multicolumn{2}{|c|}{ BDBV } & Bundibugyo ebolavirus & FDA \\
\hline \multicolumn{2}{|c|}{ TAFV } & TaïForest ebolavirus & EVD \\
\hline$\square$ & \multicolumn{2}{|c|}{$\begin{array}{l}\text { Rui Gong } \\
\text { gongr@wh.iov.cn }\end{array}$} & $\begin{array}{l}\text { RSV } \\
\text { GPt }\end{array}$ \\
\hline$\square$ & \multicolumn{2}{|c|}{$\begin{array}{l}\text { Huajun Zhang } \\
\text { hjzhang@wh.iov.cn }\end{array}$} & $\begin{array}{l}293 \mathrm{~F} \\
\mathrm{PEI}\end{array}$ \\
\hline 1 & \multicolumn{2}{|c|}{$\begin{array}{l}\text { CAS Key Laboratory of Special Pathogens and Biosafety, } \\
\text { Wuhan Institute of Virology, Center for Biosafety Mega- } \\
\text { Science, Chinese Academy of Sciences, Wuhan 430071, } \\
\text { China }\end{array}$} & $\begin{array}{l}\text { sGP } \\
\text { HA } \\
\text { CDRs }\end{array}$ \\
\hline 2 & $\begin{array}{l}\text { University } \\
\text { China }\end{array}$ & Chinese Academy of Sciences, Beijing 100049, & \\
\hline
\end{tabular}

Reston ebolavirus

Receptor binding domain

Mucin-like domain

Cleaved GP

Niemann-Pick C1

Antibody-dependent cell-mediated

cytotoxicity

Food and Drug Administration

Ebola Virus Disease

Single domain antibody

V-based single domain antibody

Respiratory syncytial virus

GP1 truncation

FreeStyle 293-F

polyethylenimine

Soluble GP

Hemagglutinin

Complementarity-determining regions 


\section{Introduction}

Ebola virus belongs to the Filoviridae family and causes the majority of hemorrhagic fever diseases, including the pandemics in West Africa during 2014-2016 which led to more than 28,600 cases and 11,325 deaths (https://www. cdc.gov/). Six species of Ebolavirus genus have been identified: Zaire ebolavirus (EBOV or ZEBOV), Sudan ebolavirus (SUDV), Bundibugyo ebolavirus (BDBV), Tai Forest ebolavirus (TAFV), Reston ebolavirus (RESTV), and Bombali ebolavirus (Emanuel et al. 2018; Karan et al. 2019). Among these, EBOV and SUDV are lethal and have been associated with large outbreaks in Africa with high human case fatalities (Negredo et al. 2011). EBOV glycoprotein (GP) is the key target of neutralizing antibodies (Lee et al. 2008). The precursor GP is cleaved by furin protease to form GP1 and GP2. These two subunits form a heterodimeric monomer through a disulfide bridge, and three GP1 form a chalice-shaped trimer, cradled by three GP2 fusion subunits. GP1 contains a receptor binding domain (RBD), which is wrapped with glycan cap region and head region, while a mucin-like domain (MLD) is linked to the side of each monomer. The GP2 subunit contains the hydrophobic internal fusion loop (IFL), two heptad repeats (HR1 and HR2), a CX6CC disulfide bond motif, a membrane-proximal external region, and a transmembrane anchor (Lee et al. 2008). After virion internalization, GP is cleaved by cathepsin B\&L to form cleaved $\mathrm{GP}\left(\mathrm{GP}_{\mathrm{CL}}\right)$ which allows binding a receptor Niemann-Pick C1 (NPC1) (Wang et al. 2016). Subsequently, the structural rearrangement of GP2 enables IFL to be exposed and inserted into the host-endosomal membrane, thereby promoting membrane fusion (Gregory et al. 2011).

Significant advances have been made towards developing potent therapeutic monoclonal antibodies (mAbs) for the treatment of EBOV infection. For example, ZMapp, a cocktail composed of three mAbs, is the first therapeutic antibody drug for emergency use in treatment of EBOVinfected patients during the 2014-EBOV outbreak in Liberia (Davey et al. 2016). Its components, 2G4 and 4G7, bind to epitopes in the GP base region and neutralize EBOV by preventing conformational rearrangements of GP during the fusion process. The third component of ZMapp, 13C6, recognizes the glycan cap region to facilitate antibody-dependent cell-mediated cytotoxicity (ADCC) (Qiu et al. 2011, 2012, 2014). Following the discovery of ZMapp, another cocktail Inmazeb (REGN-EB3) containing three mAbs developed by Regeneron Pharmaceuticals, Inc. was approved by the U.S. Food and Drug Administration (FDA) for the treatment of EBOV infection in adult and pediatric patients, including newborns of infected mothers (Markham 2021). Besides these cocktails, a human monoclonal antibody mAb114 (Ebanga) developed by Ridgeback Biotherapeutics has also been authorized by the FDA for treating EBOV infection, which was isolated from Ebola virus disease (EVD) survivors (Corti et al. 2016). MAb114 mainly binds to the GP1 core (RBD region) and still associates with it after proteolytic removal of the glycan cap, thus inhibits the binding of the cleaved GP to its receptor NPC1 (Misasi et al. 2016). In clinical trials, REGN-EB3 and mAb114 could reduce the mortality rate to $33.5 \%$ and $35.1 \%$, respectively (Mulangu et al. 2019). Although the approval of antibody drugs is a remarkable achievement in treating EBOV infection, it is still highly desirable to identify more potent neutralizing antibodies which could further decrease the mortality.

Over the past decade, great efforts have been devoted to the development of single domain antibodies (sdAbs) based on heavy chain variable domains (V-based sdAbs, V-sdAbs) such as VHHs (termed nanobodies) from heavy chains of camelid immunoglobulins (Gong and Xiao 2013). Caplacizumab is the first approved bivalent humanized nanobody targeting Von Willebrand factor for the treatment of acquired thrombotic thrombocytopenic purpura (Duggan 2018). In addition, the sdAbs show great potentials for the treatment of infectious diseases. For instance, ALX-0171 is a trivalent nanobody that can inhibit respiratory syncytial virus (RSV), which has been clinically evaluated (Detalle et al. 2016). Recently, the human IgG1 heavy chain constant domain $2(\mathrm{CH} 2)$ has been proposed as a scaffold for the development of C-based sdAbs (CsdAbs) (Dimitrov 2009; Gong and Xiao 2013). Besides the antigen-binding activity as V-sdAds, the scaffold itself might offer additional advantages since it contains binding sites or portions of binding sites for $\mathrm{Fc}$ receptors and complement $\mathrm{C} 1 \mathrm{q}$ on the framework regions, which might naturally extend the serum half-life, and mediate stability and effector functions in vivo.

In this study, we identified a novel C-sdAb M24 targeting EBOV GP that could neutralize pseudotyped and authentic EBOV in vitro. This $\mathrm{C}$-sdAb might be further developed as a therapeutic candidate against EBOV infection. Our results also provide clues for the improvement of antibody-based therapy against EVD.

\section{Materials and Methods}

\section{Expression and Purification of EBOV GP and GP Truncations}

The Codon optimized EBOV glycoprotein GP ectodomain (GPe, Zaire ebolavirus strain Mayinga, GenBank: EU224440.2) was cloned into pSectag2A vector for mammalian cellular expression. The protein lacks the 
transmembrane domain and the carboxy-terminal amino acids from 637 to 676 , with T42V/T230V mutations for the elimination of glycosylation (Lee et al. 2008). GPe $\triangle$ muc is GPe without mucin-like domain (amino acids 312-463). The C-termini of both GPe and GPe $\triangle$ muc contain $\mathrm{T} 4$ trimerization domain (Zhao et al. 2016; Agnolon et al. 2020) and StrepTagII for purification. GP1 truncation (GPt) contains residues from 54 to 222 of GPe $\triangle$ muc and carries an $\mathrm{IgG} 1 \mathrm{Fc}$ fragment at $\mathrm{C}$-terminus. The construct for sGP was truncated at residue $\mathrm{Y} 311$ and cloned into the vector pSectag2A with a 6-histidine (His) tag (Pallesen et al. 2016). The endotoxin-free plasmids were transiently transfected into FreeStyle 293-F (293F) cells using polyethylenimine (PEI) (Sigma). Five days after transfection, the supernatant was clarified by centrifugation at $8000 \times g$ for $15 \mathrm{~min}$ and passed through a $0.22 \mu \mathrm{m}$ filter. Protein sGP was purified using $\mathrm{Ni}^{2+}$-NTA Agarose (Qiagen) according to the instructions. Strep-tagged proteins were purified using Strep-Tactin ${ }^{\circledR}$ XT Superflow ${ }^{\circledR}$ (IBA) as the manufacturer's protocol. Proteins with $\mathrm{Fc}$ fragment were purified from the culture medium by protein-A sepharose (GE Healthcare). The purified proteins were concentrated using Amicon Ultra (Millipore) centrifugal filter units and were prepared in PBS.

\section{Screening of C-sdAbs Targeting EBOV GP}

A CH2-based phage display library was constructed in house as previously described (Gong et al. 2012). This library was used for panning against $\mathrm{EBOV}$ GPe $\triangle$ muc. After five rounds of panning, polyclonal phage ELISA was performed to identify candidate clones (KazemiLomedasht et al. 2015). Briefly, a 96-well plate (Corning) was coated with $2 \mu \mathrm{g} / \mathrm{mL}$ of GPe $\triangle$ muc at $4{ }^{\circ} \mathrm{C}$ overnight. BSA was incubated in the same condition as control. After blocking and washing, $10^{12} \mathrm{cfu}$ of input phage from each round after panning were added and incubated for $1 \mathrm{~h}$ at $37{ }^{\circ} \mathrm{C}$. Then wells were washed with PBS and incubated with HRP-conjugated anti-M13 (Sino Biological Inc.) for $1 \mathrm{~h}$ at $37^{\circ} \mathrm{C}$. Substrate ABTS (Life technology) was added subsequently, and the intensity of absorbance signals was measured at $405 \mathrm{~nm}$. For monoclonal phage ELISA, 200 colonies were randomly picked from the fifth round of panning, and then performed as previously reported (Gong et al. 2012). Finally, one enriched clone S5 was selected for further characterization.

\section{Expression and Purification of sdAbs}

S5 was expressed and purified following previously published protocols (Gong et al. 2012). In brief, the plasmid of S5 for expression was transformed into E. coli HB2151 bacterial culture. The expression was induced by IPTG at
$37{ }^{\circ} \mathrm{C}$ overnight. The bacterial cells were harvested and lysed by polymyxin B (Sangon) in $150 \mathrm{mmol} / \mathrm{L}$ Tris and $450 \mathrm{mmol} / \mathrm{L} \mathrm{NaCl}$ buffer. The supernatant was clarified by centrifugation and purified by $\mathrm{Ni}^{2+}$-NTA Agarose (Qiagen) according to the instructions.

\section{Expression and Purification of IgG Antibodies}

Heavy and light chains of KZ52, 13C6, 2G4 were synthesized (Sangon) according to previous reports (Lee et al. 2008; Pallesen et al. 2016) and cloned into pVITRO2-neomcs (InvivoGen). These IgGs were expressed in 293F cells and purified from the culture medium with protein A column (GE Healthcare) as described above. An EBOV neutralizing antibody mAb114 single-chain format scFv-114 with His tag and HA tag (for competitive ELISA) was cloned into pComb3XSS vector (Addgene) for expression and purification. Another version of scFv-114 with His tag and FLAG tag (for measurement of binding) was also constructed. The proteins were expressed using E. coli HB2151 and purified by $\mathrm{Ni}^{2+}$-NTA Agarose (Qiagen) as described above.

\section{Measurement of Binding}

ELISA was performed for the evaluation of binding. Briefly, 96 half-well plates (Corning) were coated overnight with $\mathrm{GPe} \triangle \mathrm{muc}$ at $4 \mu \mathrm{g} / \mathrm{mL}$. The next day, plates were washed with PBS, and then blocked for $1 \mathrm{~h}$ at $37^{\circ} \mathrm{C}$. Next, Serially-diluted antibodies were added and incubated for one hour at $37^{\circ} \mathrm{C}$. Then, 1:3000 diluted HRP-conjugated anti-flag (Sigma) was used as the secondary antibody and incubated for $1 \mathrm{~h}$ at $37{ }^{\circ} \mathrm{C}$. The intensity of absorbance signals was measured as described above. The binging ability of matured S5 (termed M24, see below) with GPe, GPt, and sGP was also tested by ELISA with the same method.

\section{Affinity Maturation}

For affinity maturation, the phage display library based on S5 with random mutations was constructed by error-prone PCR as previously described (Zhang et al. 2004). Candidate C-sdAbs were expressed as described above, and culture supernatant was used directly for binding test by ELISA.

\section{Size Exclusion Chromatography (SEC)}

Purified S5 and M24 were loaded into the Superdex 75 10/300 GL column (GE Healthcare) running on ÄKTA pure system to assess possible oligomer formation. The standard markers are BSA (67 kDa), ovalbumin (43 kDa), 
ribonuclease A (13.7 kDa), aprotinin $(6.512 \mathrm{kDa})$, and vitamin B12 (1.355 kDa). The volume of samples was 100 $\mu \mathrm{L}$ and eluted with $150 \mathrm{mmol} / \mathrm{L}$ Tris and $450 \mathrm{mmol} / \mathrm{L}$ $\mathrm{NaCl}$ buffer, $\mathrm{pH} 7.4$ at room temperature. UNICORN 7.0 software was used for data collection and analysis.

\section{Pseudovirus Neutralization Assay}

Recombinant vesicular stomatitis virus (VSV) $\mathrm{G}$ was replaced by expressing both eGFP and EBOV GPe $\triangle$ muc (rVSV-EBOV) as described previously (Wong et al. 2014). For neutralization assay, BHK-21 cells were seeded at $2.0 \times 10^{4}$ cells per well and cultured overnight in Eagle's minimal essential medium (EMEM) supplemented with $10 \%$ fetal bovine serum (FBS), 100 I.U./mL penicillin and $100 \mu \mathrm{g} / \mathrm{mL}$ streptomycin at $37{ }^{\circ} \mathrm{C}$ in an incubator containing $5 \% \quad \mathrm{CO}_{2}$. After about $24 \mathrm{~h}$, rVSV-EBOV was incubated with serial two-fold diluted S5 at an initial concentration of $5 \mu \mathrm{mol} / \mathrm{L}$ in serum-free EMEM for $1 \mathrm{~h}$ at $37{ }^{\circ} \mathrm{C}$ before infection. An EBOV neutralizing antibody mAb114 single-chain format scFv-114, and the C-sdAb scaffold were used as positive control and negative control, respectively. The amount of viruses used for infection was determined to achieve approximately $70 \%$ final infection in the control well without antibody (MOI $=0.2$ infectious units per cell). The virus-antibody mixture with equal volume was incubated with cells in EMEM supplemented with $2 \% \mathrm{FBS}$ at $37{ }^{\circ} \mathrm{C}$ and $5 \% \mathrm{CO}_{2}$ for $24 \mathrm{~h}$ before cells were fixed. Images were captured by a fluorescence microscope (Leica). The neutralization ability of M24 was also tested with the same method at the initial concentration of $1.5 \mu \mathrm{mol} / \mathrm{L}$, and the dilution was performed with a three-fold gradient. The percentages of infected cells were measured using Operetta High Content Imaging System (Perkin-Elmer). Data for neutralization were presented with the percentage of inhibition relative to control cells treated with rVSV-EBOV.

\section{Authentic Virus Neutralization Assay}

Neutralization assays involving infectious viruses were performed at the BSL-4 facility in Wuhan Institute of Virology, Chinese Academy of Sciences. Authentic EBOV (Zaire ebolavirus) was used to test the neutralization activity of M24. Vero E6 cells were seeded at $1.5 \times 10^{4}$ cells per well in the inner 60 wells of 96-well plates (Biofile) for $24 \mathrm{~h}$ prior to virus infection. Serial three-fold diluted antibodies were mixed with equal volume of authentic EBOV (1500 ffu), and incubated at $37{ }^{\circ} \mathrm{C}$ for $1 \mathrm{~h}$ before being adding to cells. Cells were fixed at $48 \mathrm{~h}$ post infection, and determined by indirect immunofluorescence assay using rabbit polyclonal antibody against EBOV NP protein (gift from Professor Zheng-Li Shi, Wuhan Institute of Virology, Chinese Academy of Science) and Alexa Fluor 488 labeled goat anti-rabbit $\operatorname{IgG}$ as the secondary antibody (Proteintech). Data were analyzed using Operetta High Content Imaging System (Perkin-Elmer) as described above.

\section{Competitive ELISA}

$\mathrm{GPe} \triangle$ muc was immobilized at $8 \mu \mathrm{g} / \mathrm{mL}$ in 96 half-well plates (Corning) at $4{ }^{\circ} \mathrm{C}$ overnight. Serial ten-fold dilutions of competitive antibodies beginning at $2 \mu \mathrm{mol} / \mathrm{L}$ were mixed with $1 \mathrm{nmol} / \mathrm{L}$ of M24 with equal volumes, and the mixture was then added to wells. HRP-conjugated anti-flag (Sigma) was used as the secondary antibody and subsequent work was performed in standard methods.

\section{Molecular Modeling and Docking}

Amino acid sequences of M24 and EBOV GP (PDB 5JQ3) (Zhao et al. 2016) in FASTA format were put into SWISSMODEL (Waterhouse et al. 2018) server as a query to search for relative protein structures. Next, data of templates were ranked according to QMEAN scoring. Topranked templates were compared and selected according to their three-dimensional structures, sequence similarity, and quaternary structural features. 3D protein model was automatically generated through the target-template alignment. Subsequently, contact and blocking residues were picked and the chosen templates were submitted to the server. Finally, ten samples with best interface-energy were returned from the server, and the docking model was chosen from the top three for analysis.

\section{Alanine Scanning}

$\mathrm{GPe} \triangle \mathrm{muc}$ mutations with alanine substitutions: E523A, I527A/G528A, I532A, and G541A were constructed and expressed using $293 \mathrm{~F}$ cells as described above. 96-well Strep-Tactin ${ }^{\circledR}$ Coated microplate (IBA) was coated with cultural supernatant of GPe $\triangle$ muc and its four mutations overnight at $4{ }^{\circ} \mathrm{C}$ respectively. The next day, diluted antibodies (scFv-114, KZ52, and C-sdAb M24) at final concentration of $200 \mathrm{nmol} / \mathrm{L}$ were added into wells for binding ELISA followed the standard method.

\section{Measurement of Binding at Low pH}

96 half-well plates (Corning) were coated with GPe $\triangle$ muc at $4{ }^{\circ} \mathrm{C}$ overnight. After blocking with $3 \%$ milk, the wells were treated with acidic buffer $(150 \mathrm{mmol} / \mathrm{L}$ Tris, $450 \mathrm{mmol} / \mathrm{L} \mathrm{NaCl}$ buffer, $\mathrm{pH}$ 5.5) for $1 \mathrm{~h}$, and with neutral buffer $(150 \mathrm{mmol} / \mathrm{L}$ Tris, $450 \mathrm{mmol} / \mathrm{L} \mathrm{NaCl}$ buffer, $\mathrm{pH}$ 7.5) as control. Next, antibodies were serially diluted with 
A

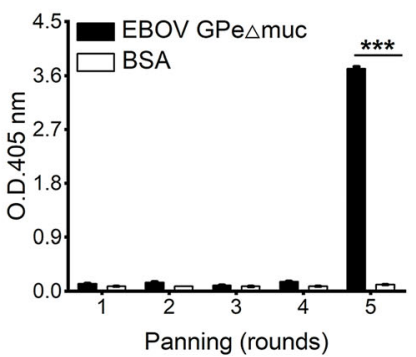

B

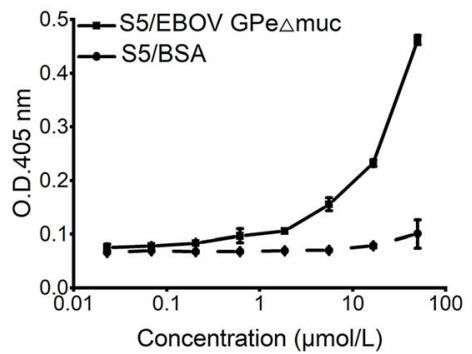

Concentration $(\mu \mathrm{mol} / \mathrm{L})$
$\mathrm{D}$

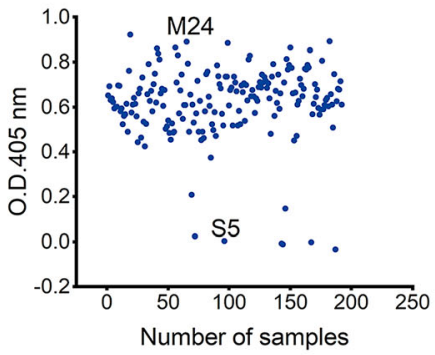

C

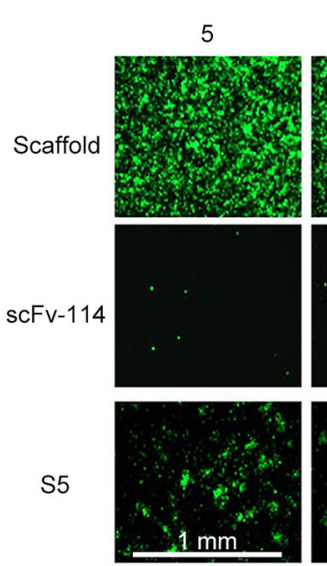

2.5
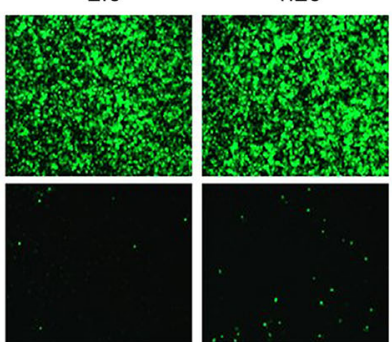

Concentration $(\mu \mathrm{mol} / \mathrm{L})$

1.25

0.6

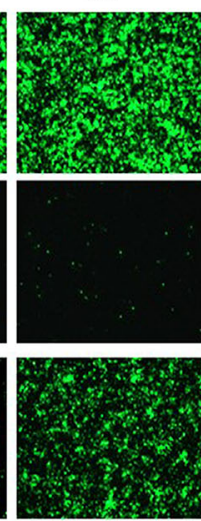

0.3

0.15
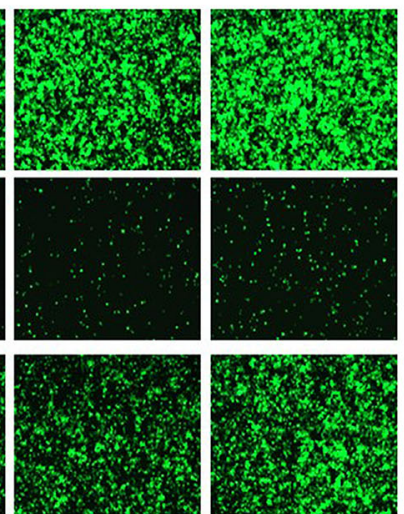

Fig. 1 Screening of C-sdAb candidates against EBOV GP. A Polyclonal phage ELISA. Phage specifically binding to EBOV GPe $\triangle$ muc (black bar) was enriched in the fifth round. Results are shown as mean \pm SEM. $* * * P<0.001$ by student's $t$-test. B Binding of $\mathrm{C}-\mathrm{sdAb} \mathrm{S} 5$ to EBOV GPe $\Delta$ muc measured by ELISA. Data are

acidic buffer and neutral buffer, respectively. Diluted antibodies were added to wells for binding ELISA as the standard method.

\section{Statistical Analysis}

All experiments were performed as triplicate. The data were expressed as mean \pm standard error of the mean (SEM). Student's $t$-test and one-way ANOVA with posthoc Tukey's test were used to compare the data from different groups. $P<0.01$ was considered statistically significant.

\section{Results}

\section{Screening of C-sdAb Candidates Against EBOV GP}

Panning against EBOV GPe $\triangle$ muc was performed from the $\mathrm{C}$-sdAb based phage display library constructed as previously reported (Gong et al. 2012). Polyclonal phage ELISA shown as mean \pm SEM. C Neutralization of S5 against replicative rVSV-EBOV. Scaffold and scFv-114 were used as negative and positive controls respectively. D Affinity maturation of S5. The single clones were screened from culture supernatant directly by ELISA.

shows the enrichment of positive clones after the fifthround panning (Fig. 1A). We then randomly picked up single colonies for the monoclonal phage ELISA. Clones with positive signals were sent for sequencing, which led to the identification of an enriched clone S5. After expression and purification, S5 exhibits specific but weak binding to EBOV GPe $\triangle$ muc (Fig. 1B). Consistent with the binding activity, S5 shows modest neutralizing activity against the pseudovirus rVSV-EBOV (Fig. 1C).

To improve neutralization potency, the affinity maturation of S5 was performed. As a result, a total of 200 specific single colonies were selected for evaluation and optimization, which resulted in the identification of one candidate clone M24 (Fig. 1D).

\section{Characterization of M24}

M24 binds to EBOV GPe $\triangle$ muc with $\mathrm{EC}_{50}$ of $0.1 \mathrm{nmol} / \mathrm{L}$ $(1.5 \mathrm{ng} / \mathrm{mL})$, which increases by over $10^{4}$ times compared to parental clone S5 (Fig. 2A). The binding activity is comparable to that of $\mathrm{scFv}-114\left(\mathrm{EC}_{50}\right.$ of $0.4 \mathrm{nmol} / \mathrm{L}$ or 
Fig. 2 Characterization of purified C-sdAb M24 against EBOV GP. A Binding activity of M24 (matured C-sdAb S5) to EBOV GPe $\Delta$ muc measured by ELISA. B Binding activity of scFv-114 to EBOV GPe $\Delta$ muc measured by ELISA. ELISA data are shown as mean \pm SEM. C Evaluation of oligomer formation of M24 and S5 measured by SEC. A standard curve was determined based on the elution volumes of the protein standards. 1. BSA, $67 \mathrm{kDa}$; 2. Ovalbumin, $43 \mathrm{kDa}$; 3. Ribonuclease A, $13.7 \mathrm{kDa} ; 4$. Aprotinin, $6.512 \mathrm{kDa} ; 5$. Vitamin, $1.355 \mathrm{kDa}$.
A
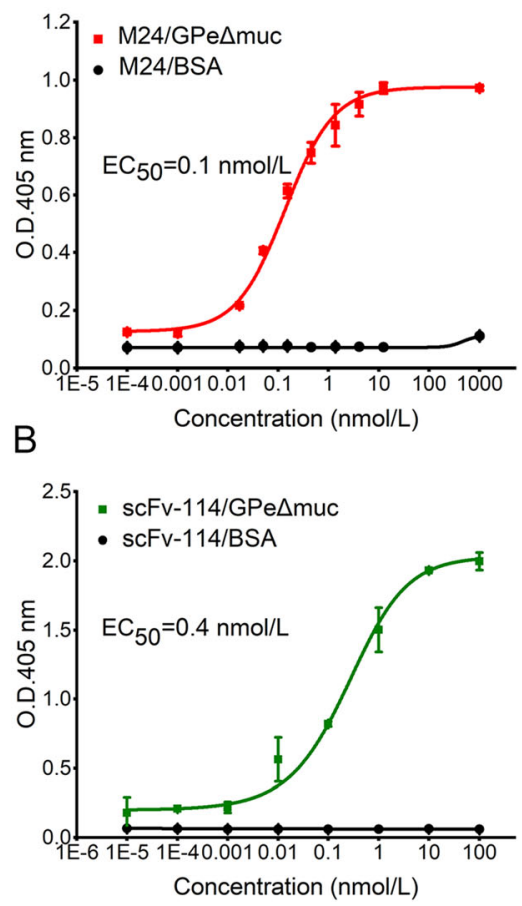

C

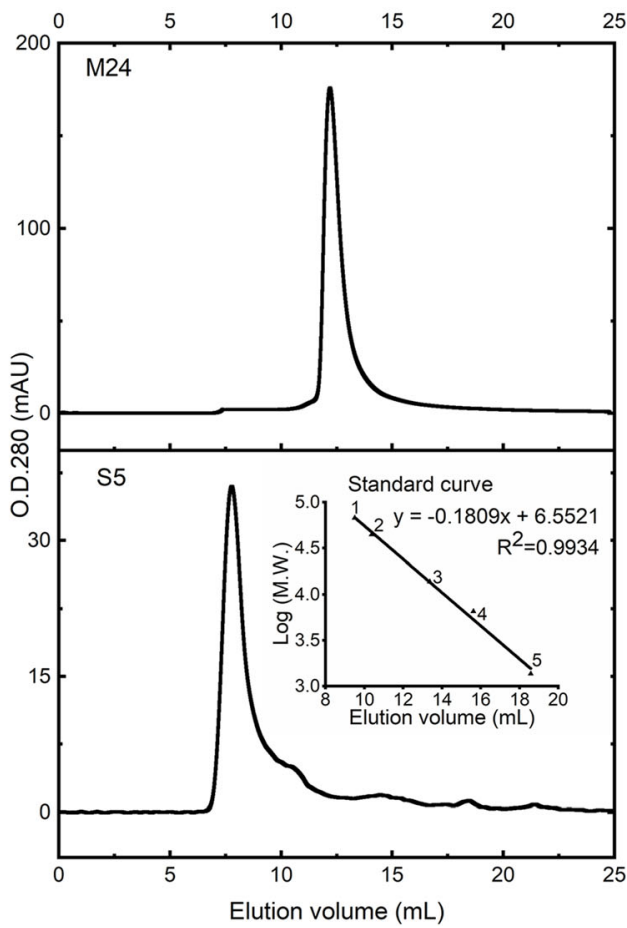

$10 \mathrm{ng} / \mathrm{mL}$ ) (Fig. 2B). Meanwhile, M24 exists as a monomer, whereas S5 forms an oligomer in the solution (Fig. 2C). Therefore, the affinity maturation process not only increases affinity, but also improves the physicochemical property.

We then tested the neutralization ability of M24 against rVSV-EBOV. As shown in Fig. 3A, M24 effectively neutralized $\mathrm{rVSV}-\mathrm{EBOV}$ with $\mathrm{IC}_{50}$ of $0.8 \mathrm{nmol} / \mathrm{L}(12 \mathrm{ng} / \mathrm{mL})$. Similarly, the positive control scFv-114 inhibited rVSVEBOV with $\mathrm{IC}_{50}$ of $2.8 \mathrm{nmol} / \mathrm{L}(70 \mathrm{ng} / \mathrm{mL})$, which was consistent with the previous study (Corti et al. 2016).

The neutralization activity of M24 was then further evaluated with authentic EBOV (Zaire ebolavirus). We found that M24 modestly neutralized authentic EBOV with a maximum inhibition efficacy of $31.6 \%$. By contrast, scFv-114 could efficiently neutralize authentic EBOV (Fig. 3B). Since M24 strongly neutralized the pseudotyped EBOV but weakly inhibited live EBOV, we tried to perform more studies to find out the possible reasons.

\section{Identification of the Putative M24 Epitope}

To determine the epitope recognized by M24, we constructed different GP truncations (Fig. 4A) and evaluated the binding of M24 to them. Firstly, we found that M24 could still efficiently bind to GPe with $\mathrm{EC}_{50}$ of $4.8 \mathrm{nmol} / \mathrm{L}$ (72 ng/mL) and scFv-114 could bind to GPe with $\mathrm{EC}_{50}$ of $5.0 \mathrm{nmol} / \mathrm{L}$ (125 ng/mL) (Fig. 4B). However, their binding to GPe significantly decreased compared to their binding to
GPe $\triangle$ muc. One concern is that although MLD is dispensable for M24 binding, it may hinder the binding of M24, thus leading to the modest neutralization of M24 against live EBOV. However, scFv-114 also significantly lost the binding to GPe since the coverage of RBD by MLD, but it efficiently neutralized live EBOV due to tightly binding to $\mathrm{GP}_{\mathrm{CL}}$. Therefore, the loss of binding of M24 to GPe might not be the major reason for the weak inhibition against live EBOV. Next, a GP truncation was tested to determine the binding regions for M24. GPt is a truncated GP1 containing RBD but lacks glycan cap and MLD (Fig. 4A). M24 and the glycan cap binder 13C6 could not bind to GPt, while scFv-114, the RBD binder, bound to the GPt (Fig. 4C). M24 and 2G4 could not bind to soluble GP (sGP), a sGP that shares 295 residues with the GPe (Fig. 4A), while 13C6 bound strongly to it (Fig. 4D). These data indicate that MLD and RBD are unnecessary for the binding of M24 to GP, and M24 does not bind to sGP alone.

To confirm these findings, we further performed a competitive ELISA. M24 had no competition with the glycan cap binder $13 \mathrm{C} 6$, the RBD binder mAb114, or the base binder KZ52 and 2G4 (Fig. 4E). These results indicate that the epitope of M24 is distinct from the antibodies tested in this study.

To refine the epitope of M24, we firstly used molecular modeling and docking for prediction. The computationally predicated GPe/M24 complex indicated that M24 might recognize residues in the IFL region of GP2, which differed 
A rVSV-EBOV

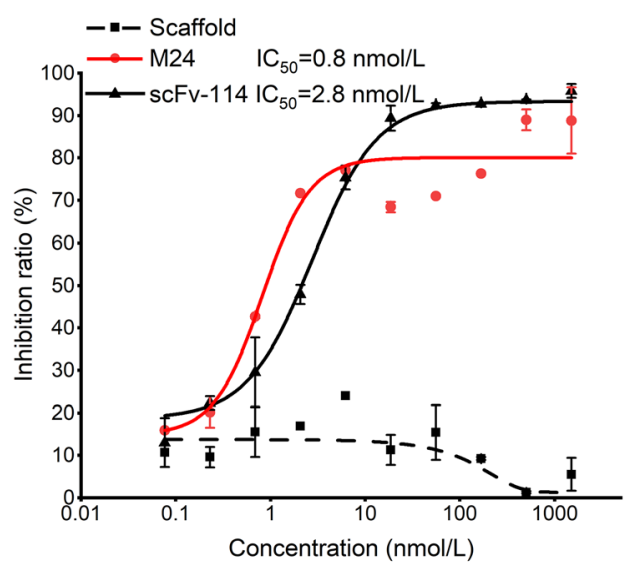

B

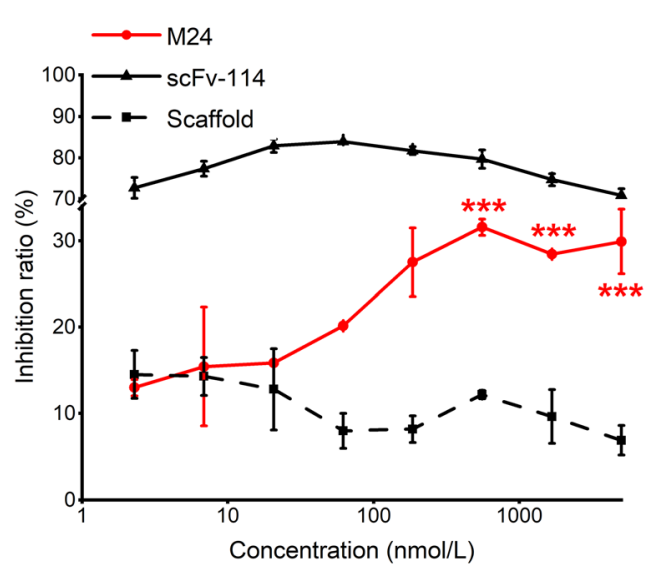

Fig. 3 Neutralization of M24 against rVSV-EBOV (A) and authentic EBOV (B). Scaffold and scFv-114 were used as negative and positive controls respectively. Data for neutralization are shown in the percentage of inhibition relative to control cells infected by EBOV alone. Results are shown as mean \pm SEM, $* * * P<0.001$ by one-way ANOVA with Tukey's post hoc test.

from KZ52 and mAb114 (Fig. 5A). To refine the critical residues in GP for M24 binding, we next evaluated the binding of M24 against GP mutations, including GP E523A, GP I527A\&G528A, GP I532A, and GP G541A (Fig. 5B) in the IFL of GP2 according to the results of docking. As a result, the binding of M24 to these mutations has dramatically decreased compared to its binding to wildtype GP. In contrast, the binding of mAb114 and KZ52 to these mutants was unaffected (Fig. 5C). Notably, alanine substitution in 1532 residue reduced the binding of KZ52 and M24 by $54.7 \%$ and $73.2 \%$ respectively but did not affect the mAb114 binding. Overall, these epitope mapping analyses indicate that residues E523, I527\&G528, I532, and G541 within IFL of GP2 may be critical for M24 binding. What's more, these four residues are highly conserved among GPs of Zaire ebolavirus (Fig. 5D). In conclusion, these results prove further evidence that IFL is an effective target for the development of anti-EBOV agents.

\section{Binding of M24 to GP at Acidic pH}

Receptor interaction and membrane fusion of filovirus occur in the acidified endosomes, and acidic $\mathrm{pH}$ could shift the GP2 conformational equilibrium to an intermediate state in favor of NPC1 binding (Das et al. 2020). We next examined whether M24 could bind to GP at acidic pH by ELISA (Fig. 6). As a result, M24 binds to GPe $\triangle$ muc with $\mathrm{EC}_{50}$ of $9.6 \mathrm{nmol} / \mathrm{L}(144 \mathrm{ng} / \mathrm{mL})$ at $\mathrm{pH} 5.5$, which has been decreased about 100 times than the binding at natural $\mathrm{pH}$ (Fig. 6A). However, the binding of scFv-114 and KZ52 is independent of $\mathrm{pH}$ (Fig. 6B and 6C). Therefore, the decrease of binding to GP at acidic condition might be a crucial factor for the weak neutralization activity of M24 against authentic EBOV.

\section{Discussion}

The current antibody-based treatment for EVD has indeed been an unprecedented success. However, over $30 \%$ of antibody-treated patients still died of high viral loads according to clinical trials. Hence, better effective antiEBOV agents are required to meet more medical needs.

Since all approved antibodies against EBOV infection are full-length format currently, we have attempted to use the sdAb technique platform to select potential candidates. A great deal of work has been devoted towards the development of binding domains based on novel scaffolds from altered protein folding domains with much smaller size compared to conventional full-length antibodies. The sdAbs are quite attractive candidates for the treatment of various diseases, including infectious diseases (Schepens et al. 2011; Wu et al. 2020). However, to our knowledge, no sdAb against EBOV infection has been reported. In our previous work, the antibody $\mathrm{CH} 2$ domain was engineered to make it more suitable as a scaffold for the development of C-sdAbs (Gong et al. 2009, 2011; Gao et al. 2019; Cao et al. 2020) and showed the potentials of functional C-sdAbs as therapeutic candidates (Xiao et al. 2009; Gong et al. 2012). In this study, we screened $\mathrm{C}$-sdAb phage display libraries against EBOV GP and identified one $\mathrm{C}$-sdAb that has shown strong neutralizing activity against pseudotyped EBOV but modest inhibition to authentic EBOV. To further reveal the mechanism of the neutralization, we performed experiments to refine the epitope.

Based on the truncated GP binding analysis and computational prediction, we found that the binding region of M24 is located within the IFL of GP2. A mouse-derived 
Fig. 4 Binding region determination. A Construction of different EBOV GP truncations. GPe includes receptor-binding domain (RBD, 54-201), Glycan cap (227-310), mucin-like domain (MLD, 314-462), internal fusion loop (IFL, 511-556). GP truncation $(\mathrm{GPt})$ is from 55 to 222 . $\mathrm{sGP}$ is from 33-311 residues. B-

D Binding of M24 and different control antibodies to different GP constructs. E Competitive ELISA between M24 and scFv114, KZ52, 13C6, and 2G4. Biotinylated-M24 (M24-bio) was a positive control. All data are shown as mean \pm SEM.
A

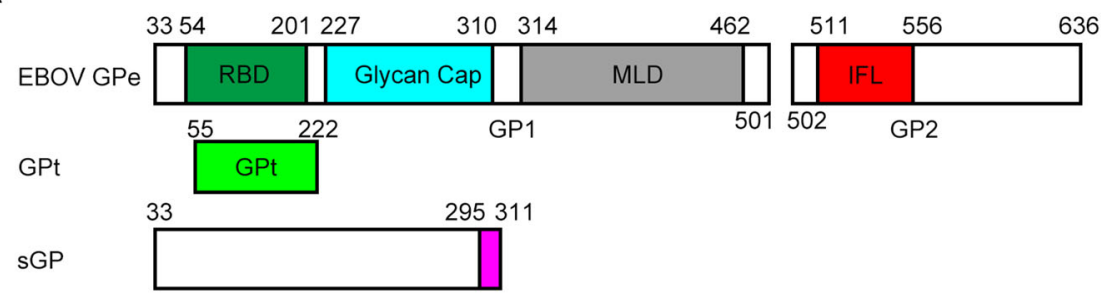

B

$\mathrm{GPe}$

C

GPt
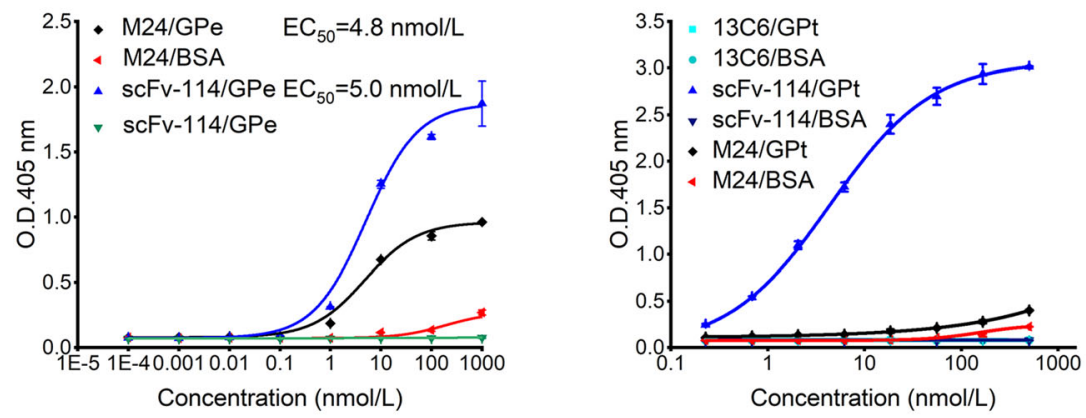

D

sGP

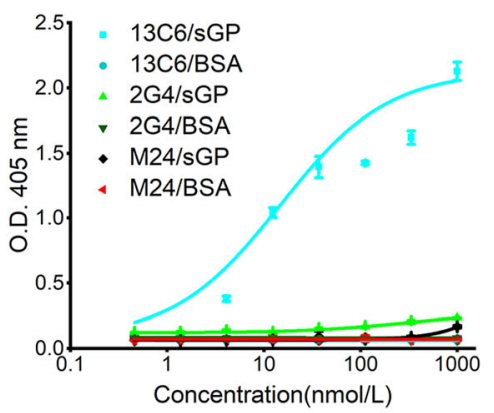

E Competitive ELISA

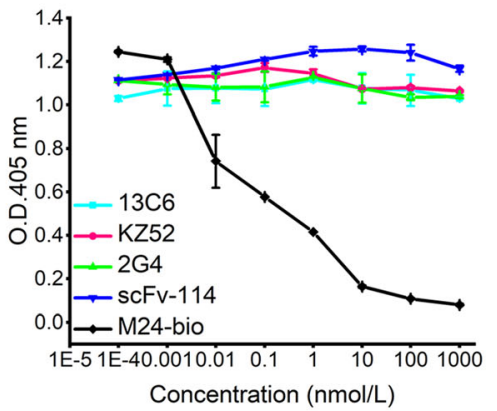

$\mathrm{mAb}$ 6D6 and a macaque-derived mAb CA45 recognize the IFL (Zhao et al. 2017; Milligan et al. 2019). Interestingly, the epitopes of these identified antibodies targeting IFL more or less span the base region that shares with the epitope of KZ52. In contrast, M24 appears to be a novel epitope targeting IFL without spanning the base region according to the result of competitive ELISA. Notably, it has been pointed out that the contact of IFL-targeting antibodies to residues in both IFL and GP1 is essential for neutralizing activity (Wec et al. 2017; Zhao et al. 2017; Milligan et al. 2019). In consistence with this point, FVM02, an IFL mAb that binds to the tip of IFL with no GP1 contact lacks neutralizing activity in vitro although it could provide $30 \%$ protection in mice due to $\mathrm{Fc}$-mediated effector functions (Keck et al. 2016). Despite the low efficacy, M24 targeting the IFL epitope without GP1 contact still has neutralizing activity in vitro. The difference may provide novel understanding of the IFL epitope. In addition, M24 recognizing epitope is relatively conserved among Zaire ebolavirus, which could be a broad-spectrum target for drug and vaccine development.

When the virion is internalized, the GP2 is rearranged to form a fusion core termed the six-helix bundle, which promotes membrane fusion in the endosome under an acidic condition. In this process, a low-pH condition triggers a reversible conformational change in GP, which results in the state for NPC1 binding (Wang et al. 2016; Das et al. 2020). Under acidic $\mathrm{pH}$, antibodies such as mAb114 and CA45 remain the binding ability to GP (Misasi et al. 2016; Zhao et al. 2017), suggesting that reservation of GP binding in the acidic condition may play a significant role in neutralizing activity. On the contrary, the decreased binding of M24 under the acidic condition probably attributes to some uncertain conformational changes caused by instability of itself, which leads to modest neutralizing activity against authentic EBOV. These findings suggest that further engineering of M24 for the increase of its stability, including the 


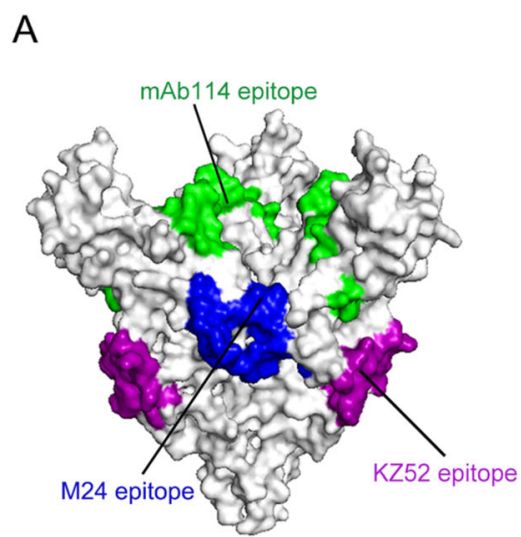

B

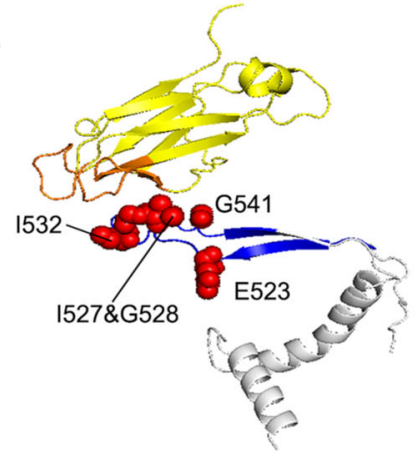

C

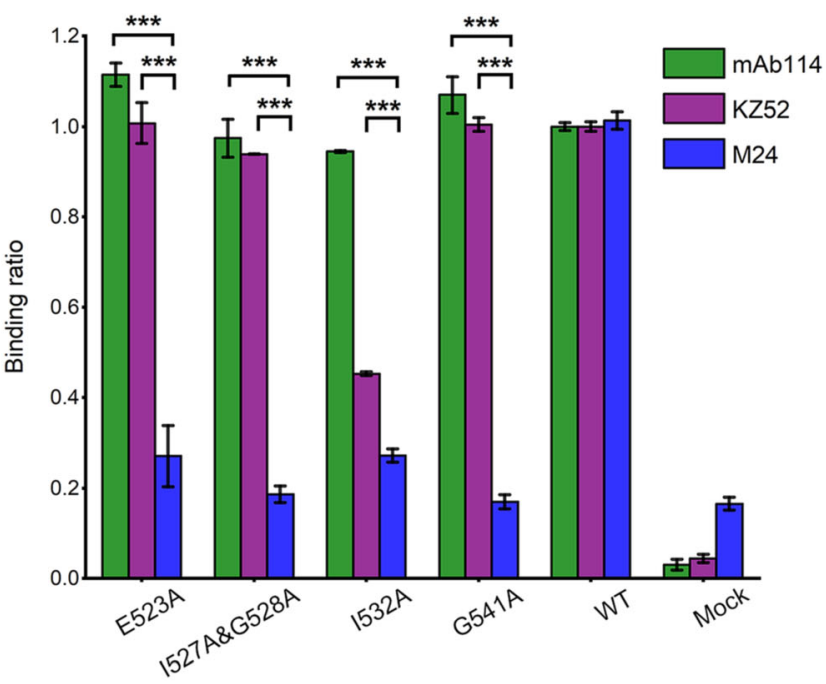

D

$523 \quad 527 \& 528 \quad 532 \quad 541$

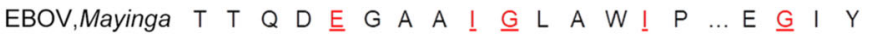
EBOV,Kikwit T T Q D E G A A I G L A W I P SUDV,Boniface $T A Q$ E $\underline{Q} H N A \underline{A} \underline{G}|A \quad W \underline{l} P \ldots E \underline{G}| Y$

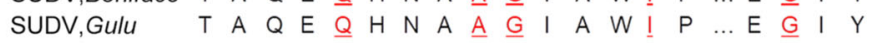
BDBV $\quad T \quad T Q D \underline{E} G A A \underline{G} L A N \underline{G} \quad P E \underline{G} \mid Y$ TAFV T A L D $\underline{E} G A A \underline{G} L A N \underline{I} P \ldots E \underline{G} \mid Y$ RESTV $\quad T A \vee D E G A A \underline{G} L A \quad W \quad P \quad F E \underline{G} \mid Y$

Fig. 5 Epitope mapping and analysis of M24. A Epitope presentation in trimeric EBOV GPe $\triangle$ muc. Predicted M24 epitope, blue; KZ52 epitope, purple; mAb114 epitope, green. B M24 and GP2 complex by molecular docking. M24 scaffold is colored yellow and its binding regions are colored orange. IFL in GP is colored blue. The critical residues (E523, I527\&G528, I532, and G541) in IFL involved in binding are shown in red spheres. Other GP regions are colored grey. C Binding of M24 (blue) to different GP mutants. All the mutants could still bind to mAb114 (green) while only one mutation affects the binding to KZ52 (purple). Mock reflects GP binding of untransfected cell supernatant. Results are shown as mean \pm SEM. *** $P<0.001$ by one-way ANOVA with Tukey's post hoc test. D Sequence alignment of IFL regions in different EBOV GPs. In general, these five residues (red) are conserved while variations have been found between EBOV and SUDV.
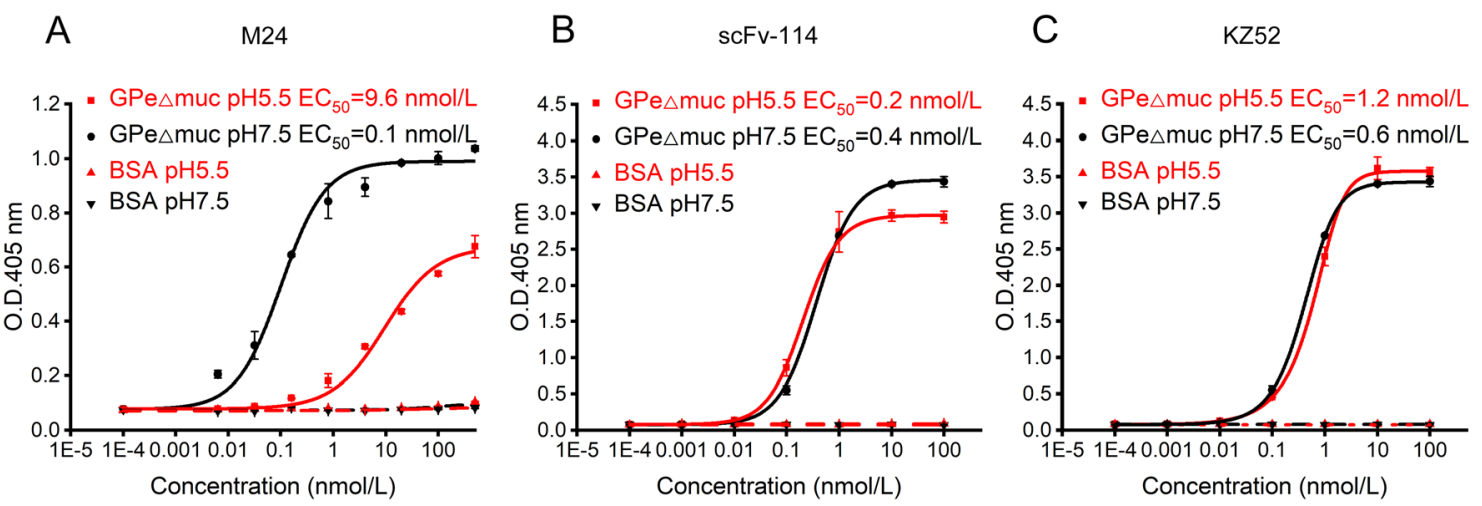

Fig. 6 Binding of M24 (A), scFv-114 (B), and KZ52 (C) to $\mathrm{GPe} \triangle \mathrm{muc}$ at neutral and acidic $\mathrm{pH}$ conditions. In the case of M24, the binding at $\mathrm{pH} 5.5$ (red) decreases dramatically compared to the

stabilization of the complementarily-determining regions (CDRs) may lead to higher binding affinity to EBOV GP binding at $\mathrm{pH} 7.5$ (black). In contrast, the binding of $\mathrm{scFv}-114$ and KZ52 at pH 5.5 (red) has no significant change compared to the binding at $\mathrm{pH} 7.5$ (black). Data are shown as mean \pm SEM.

under both acidic and neutral conditions, thus may increase the neutralizing efficacy. 
In summary, we identified and characterized a novel C-sdAb M24 against EBOV GP, a potential inhibitor for neutralizing EBOV. It offers a proof-of-concept that sdAbs could be therapeutic candidates against EBOV infections. Also, our findings provide useful epitope information on functional epitopes for the development of anti-EBOV drugs and vaccines, which might strengthen our capacity to tackle the EBOV outbreak in the future.

Acknowledgements We thank the Core Facility and Technical Support, Wuhan Institute of Virology, Chinese Academy of Sciences; Wuhan Institute of Biotechnology; Wuhan Key Laboratory on Emerging Infectious Diseases and Biosafety; Center for Biosafety Mega-Science, Chinese Academy of Sciences; and the National Virus Resource Center for resource support. We are grateful to the Wuhan National Biosafety Laboratory support team, including the engineering, biosafety, biosecurity, and administrative staff. This work was jointly supported by the Advanced Customer Cultivation Project of Wuhan National Biosafety Laboratory, Chinese Academy of Sciences (2021ACCP-MS01, 2019ACCP-ZD03), the Natural Science Foundation of Hubei Province of China (2019CFA076), and the National Natural Science Foundation of China (31870926).

Author Contributions RG contributed to the conception of the study, data analysis, and manuscript preparation. RW performed the experiment, analyzed data and wrote the manuscript. HZ, CP, and JS helped to perform the experiment. HZ helped experimental analysis with constructive discussions. RG finalized the manuscript. All authors read and approved the final version of the manuscript.

\section{Compliance with Ethical Standards}

Conflict of interest The authors declare that they have no conflicts of interest with the contents of this article.

Animal and Human Rights Statement This article does not contain any studies with human or animal subjects performed by any of the authors.

\section{References}

Agnolon V, Kiseljak D, Wurm MJ, Wurm FM, Foissard C, Gallais F, Wehrle S, Muñoz-Fontela C, Bellanger L, Correia BE, Corradin G, Spertini F (2020) Designs and characterization of subunit Ebola GP vaccine candidates: implications for immunogenicity. Front Immunol 11:586595

Cao G, Gao X, Zhan Y, Wang Q, Zhang Z, Dimitrov DS, Gong R (2020) An engineered human $\mathrm{IgG} 1 \mathrm{CH} 2$ domain with decreased aggregation and nonspecific binding. Mabs 12:1689027

Corti D, Misasi J, Mulangu S, Stanley DA, Kanekiyo M, Wollen S, Ploquin A, Doria-Rose NA, Staupe RP, Bailey M, Shi W, Choe M, Marcus H, Thompson EA, Cagigi A, Silacci C, FernandezRodriguez B, Perez L, Sallusto F, Vanzetta F, Agatic G, Cameroni E, Kisalu N, Gordon I, Ledgerwood JE, Mascola JR, Graham BS, Muyembe-Tamfun JJ, Trefry JC, Lanzavecchia A, Sullivan NJ (2016) Protective monotherapy against lethal Ebola virus infection by a potently neutralizing antibody. Science 351:1339-1342

Das DK, Bulow U, Diehl WE, Durham ND, Senjobe F, Chandran K, Luban J, Munro JB (2020) Conformational changes in the Ebola virus membrane fusion machine induced by $\mathrm{pH}, \mathrm{Ca} 2+$, and receptor binding. PLoS Biol 18:e3000626

Davey RT, Dodd L, Proschan MA, Neaton J, Neuhaus Nordwall J, Koopmeiners JS, Beigel J, Tierney J, Lane HC, Fauci AS, Massaquoi MBF, Sahr F, Malvy D (2016) A randomized, controlled trial of ZMapp for Ebola virus infection. N Engl J Med 375:1448-1456

Detalle L, Stohr T, Palomo C, Piedra PA, Gilbert BE, Mas V, Millar A, Power UF, Stortelers C, Allosery K, Melero JA, Depla E (2016) Generation and characterization of ALX-0171, a potent novel therapeutic nanobody for the treatment of respiratory syncytial virus infection. Antimicrob Agents Chemother 60:6-13

Dimitrov DS (2009) Engineered $\mathrm{CH} 2$ domains (nanoantibodies). Mabs 1:26-28

Duggan S (2018) Caplacizumab: First Global Approval. Drugs 78:1639-1642

Emanuel J, Marzi A, Feldmann H (2018) Filoviruses: ecology, molecular biology, and evolution. Adv Virus Res 100:189-221

Gao X, Conard A, Yang C, Zhan Y, Zeng F, Shi J, Li W, Dimitrov DS, Gong R (2019) Optimization of the C-terminus of an autonomous human IgG1 $\mathrm{CH} 2$ domain for stability and aggregation resistance. Mol Pharm 16:3647-3656

Gong R, Xiao G (2013) Engineered antibody variable and constant domains as therapeutic candidates. Pharm Pat Anal 2:637-646

Gong $\mathrm{R}, \mathrm{Vu} \mathrm{BK}$, Feng Y, Prieto DA, Dyba MA, Walsh JD, Prabakaran P, Veenstra TD, Tarasov SG, Ishima R, Dimitrov DS (2009) Engineered human antibody constant domains with increased stability. J Biol Chem 284:14203-14210

Gong R, Wang Y, Feng Y, Zhao Q, Dimitrov DS (2011) Shortened engineered human antibody $\mathrm{CH} 2$ domains: increased stability and binding to the human neonatal $\mathrm{Fc}$ receptor. J Biol Chem 286:27288-27293

Gong R, Wang Y, Ying T, Dimitrov DS (2012) Bispecific engineered antibody domains (nanoantibodies) that interact noncompetitively with an HIV-1 neutralizing epitope and FcRn. PLoS ONE 7:e42288

Gregory SM, Harada E, Liang B, Delos SE, White JM, Tamm LK (2011) Structure and function of the complete internal fusion loop from Ebolavirus glycoprotein 2. Proc Natl Acad Sci USA 108:11211-11216

Karan LS, Makenov MT, Korneev MG, Sacko N, Boumbaly S, Yakovlev SA, Kourouma K, Bayandin RB, Gladysheva AV, Shipovalov AV, Yurganova IA, Grigorieva YE, Fedorova MV, Scherbakova SA, Kutyrev VV, Agafonov AP, Maksyutov RA, Shipulin GA, Maleev VV, Boiro M, Akimkin VG, Popova AY (2019) Bombali virus in mops condylurus bats, Guinea. Emerg Infect Dis 25:1774-1775

Kazemi-Lomedasht F, Behdani M, Bagheri KP, Habibi-Anbouhi M, Abolhassani M, Arezumand R, Shahbazzadeh D, Mirzahoseini H (2015) Inhibition of angiogenesis in human endothelial cell using VEGF specific nanobody. Mol Immunol 65:58-67

Keck Z-Y, Enterlein SG, Howell KA, Vu H, Shulenin S, Warfield KL, Froude JW, Araghi N, Douglas R, Biggins J, Lear-Rooney CM, Wirchnianski AS, Lau P, Wang Y, Herbert AS, Dye JM, Glass PJ, Holtsberg FW, Foung SKH, Aman MJ (2016) Macaque monoclonal antibodies targeting novel conserved epitopes within filovirus glycoprotein. J Virol 90:279-291

Lee JE, Fusco ML, Hessell AJ, Oswald WB, Burton DR, Saphire EO (2008) Structure of the Ebola virus glycoprotein bound to an antibody from a human survivor. Nature 454:177-182

Markham A (2021) REGN-EB3: first approval. Drugs 81:175-178

Milligan JC, Parekh DV, Fuller KM, Igarashi M, Takada A, Saphire EO (2019) Structural characterization of pan-Ebolavirus antibody 6D6 targeting the fusion peptide of the surface glycoprotein. J Infect Dis 219:415-419 
Misasi J, Gilman MS, Kanekiyo M, Gui M, Cagigi A, Mulangu S, Corti D, Ledgerwood JE, Lanzavecchia A, Cunningham J, Muyembe-Tamfun JJ, Baxa U, Graham BS, Xiang Y, Sullivan NJ, McLellan JS (2016) Structural and molecular basis for Ebola virus neutralization by protective human antibodies. Science 351:1343-1346

Mulangu S, Dodd LE, Davey RT Jr, Tshiani Mbaya O, Proschan M, Mukadi D, Lusakibanza Manzo M, Nzolo D, Tshomba Oloma A, Ibanda A, Ali R, Coulibaly S, Levine AC, Grais R, Diaz J, Lane HC, Muyembe-Tamfum JJ, Group PW, Sivahera B, Camara M, Kojan R, Walker R, Dighero-Kemp B, Cao H, Mukumbayi P, Mbala-Kingebeni P, Ahuka S, Albert S, Bonnett T, Crozier I, Duvenhage M, Proffitt C, Teitelbaum M, Moench T, Aboulhab J, Barrett K, Cahill K, Cone K, Eckes R, Hensley L, Herpin B, Higgs E, Ledgerwood J, Pierson J, Smolskis M, Sow Y, Tierney J, Sivapalasingam S, Holman W, Gettinger N, Vallee D, Nordwall J, Team PCS (2019) A randomized, controlled trial of ebola virus disease therapeutics. N Engl J Med 381:2293-2303

Negredo A, Palacios G, Vázquez-Morón S, González F, Dopazo H, Molero F, Juste J, Quetglas J, Savji N, de la Cruz Martínez M, Herrera JE, Pizarro M, Hutchison SK, Echevarría JE, Lipkin WI, Tenorio A (2011) Discovery of an ebolavirus-like filovirus in europe. PLoS Pathog 7:e1002304

Pallesen J, Murin CD, de Val N, Cottrell CA, Hastie KM, Turner HL, Fusco ML, Flyak AI, Zeitlin L, Crowe JE Jr, Andersen KG, Saphire EO, Ward AB (2016) Structures of Ebola virus GP and sGP in complex with therapeutic antibodies. Nat Microbiol $1: 16128$

Qiu X, Alimonti JB, Melito PL, Fernando L, Stroher U, Jones SM (2011) Characterization of Zaire ebolavirus glycoprotein-specific monoclonal antibodies. Clin Immunol 141:218-227

Qiu X, Audet J, Wong G, Pillet S, Bello A, Cabral T, Strong JE, Plummer F, Corbett CR, Alimonti JB, Kobinger GP (2012) Successful treatment of ebola virus-infected cynomolgus macaques with monoclonal antibodies. Sci Transl Med 4:138ra181

Qiu X, Wong G, Audet J, Bello A, Fernando L, Alimonti JB, Fausther-Bovendo H, Wei H, Aviles J, Hiatt E, Johnson A, Morton J, Swope K, Bohorov O, Bohorova N, Goodman C, Kim D, Pauly MH, Velasco J, Pettitt J, Olinger GG, Whaley K, Xu B, Strong JE, Zeitlin L, Kobinger GP (2014) Reversion of advanced Ebola virus disease in nonhuman primates with ZMapp. Nature 514:47-53

Schepens B, Ibanez LI, De Baets S, Hultberg A, Bogaert P, De Bleser P, Vervalle F, Verrips T, Melero J, Vandevelde W, Vanlandschoot P, Saelens X (2011) Nanobodies(R) specific for respiratory syncytial virus fusion protein protect against infection by inhibition of fusion. J Infect Dis 204:1692-1701

Wang H, Shi Y, Song J, Qi J, Lu G, Yan J, Gao GF (2016) Ebola viral glycoprotein bound to its endosomal receptor Niemann-Pick C1. Cell 164:258-268

Waterhouse A, Bertoni M, Bienert S, Studer G, Tauriello G, Gumienny R, Heer FT, de Beer TAP, Rempfer C, Bordoli L, Lepore R, Schwede T (2018) SWISS-MODEL: homology modelling of protein structures and complexes. Nucleic Acids Res 46:W296-W303

Wec AZ, Herbert AS, Murin CD, Nyakatura EK, Abelson DM, Fels JM, He S, James RM, de La Vega M-A, Zhu W, Bakken RR, Goodwin E, Turner HL, Jangra RK, Zeitlin L, Qiu X, Lai JR, Walker LM, Ward AB, Dye JM, Chandran K, Bornholdt ZA (2017) Antibodies from a human survivor define sites of vulnerability for broad protection against ebolaviruses. Cell 169

Wong G, Audet J, Fernando L, Fausther-Bovendo H, Alimonti JB, Kobinger GP, Qiu X (2014) Immunization with vesicular stomatitis virus vaccine expressing the Ebola glycoprotein provides sustained long-term protection in rodents. Vaccine 32:5722-5729

Wu Y, Li C, Xia S, Tian X, Kong Y, Wang Z, Gu C, Zhang R, Tu C, Xie Y, Yang Z, Lu L, Jiang S, Ying T (2020) Identification of human single-domain antibodies against SARS-CoV-2. Cell Host Microbe 27:891-898 e895

Xiao X, Feng Y, Vu BK, Ishima R, Dimitrov DS (2009) A large library based on a novel (CH2) scaffold: identification of HIV-1 inhibitors. Biochem Biophys Res Commun 387:387-392

Zhang MY, Shu Y, Rudolph D, Prabakaran P, Labrijn AF, Zwick MB, Lal RB, Dimitrov DS (2004) Improved breadth and potency of an HIV-1-neutralizing human single-chain antibody by random mutagenesis and sequential antigen panning. J Mol Biol 335:209-219

Zhao Y, Ren J, Harlos K, Jones DM, Zeltina A, Bowden TA, PadillaParra S, Fry EE, Stuart DI (2016) Toremifene interacts with and destabilizes the Ebola virus glycoprotein. Nature 535:169-172

Zhao X, Howell KA, He S, Brannan JM, Wec AZ, Davidson E, Turner HL, Chiang C-I, Lei L, Fels JM, Vu H, Shulenin S, Turonis AN, Kuehne AI, Liu G, Ta M, Wang Y, Sundling C, Xiao Y, Spence JS, Doranz BJ, Holtsberg FW, Ward AB, Chandran K, Dye JM, Qiu X, Li Y, Aman MJ (2017) Immunization-elicited broadly protective antibody reveals ebolavirus fusion loop as a site of vulnerability. Cell 169:891-904.e15 